\title{
Novel insights into the role of hypoxia-inducible factor-1 in the pathogenesis of human post-intubation tracheal stenosis
}

\author{
ZHIGANG CAI ${ }^{1}$, HAITAO LI $^{1}$, HEFANG ZHANG $^{2}$, SHUO HAN $^{3}$, RUIJIN AN $^{3}$ and XIXIN YAN ${ }^{1}$ \\ ${ }^{1}$ Department of Respiratory Medicine, The Second Hospital of Hebei Medical University, Shijiazhuang, \\ Hebei 050000; ${ }^{2}$ Department of Endocrinology, The Traditional Chinese Hospital of Hebei Medical University; \\ ${ }^{3}$ Department of Anatomy, Hebei Medical University, Shijiazhuang, Hebei 050011, P.R. China
}

Received March 20, 2013; Accepted July 18, 2013

DOI: $10.3892 / \mathrm{mmr} .2013 .1595$

\begin{abstract}
Hypoxia is an important mechanism involved in dermal scar formation. Post-intubation tracheal stenosis (PTS) has similar pathological characteristics and formation mechanisms to skin hypertrophic scars. Hypoxia-inducible factor- $1 \alpha$ (HIF-1 $\alpha$ ) is a nuclear transcription factor that facilitates the adaptation of human cells to compromised oxygen tension under hypoxic conditions. The aim of this study was to investigate whether hypoxia and HIF-1 $\alpha$ were involved in PTS. In the first part of our study, we observed the effects of tracheal mucosal pressure exerted by the endotracheal tube cuff and duration of intubation on tracheal stenosis using prospective methods. In the second part of our study, 24 patients were divided into three groups, according to the characteristics observed using bronchoscopy: granulation phase group, proliferative phase group and mature phase group. Tissues showing dysplasia were obtained using bronchoscopy forceps, and western blotting was performed to detect the protein expression of HIF- $1 \alpha$ and its target genes, including vascular endothelial growth factor (VEGF), basic fibroblast growth factor (bFGF) and transforming growth factor (TGF)- $\beta$. In experiment one, we observed that the tracheal mucosal pressure exerted by the endotracheal tube cuff in the PTS group was significantly higher compared with that in the group without PTS, and the duration of intubation was longer compared with that in the group without PTS $(\mathrm{P}<0.05)$. In experiment two, we observed that the expression of HIF-1 $\alpha$ was highest in the granulation phase, showed a decrease in the proliferative phase and in the mature phase was significantly reduced compared with the other two phases. The results were similar for VEGF and bFGF. TGF- $\beta$ protein expression was highest in the proliferative phase, significantly reduced in the mature phase and the same trends were observed for collagen type I and III, and
\end{abstract}

Correspondence to: Professor Xixin Yan, Department of Respiratory Medicine, The Second Hospital of Hebei Medical University, Shijiazhuang, Hebei 050000, P.R. China

E-mail: caibin518@sina.com

Key words: tracheal stenosis, hypoxia-inducible factor-1, growth factor, fibroblast, activation $\alpha$-smooth muscle actin. HIF-1 $\alpha$ is involved in the pathogenesis of PTS and may be a potential key regulator in the initiation and facilitation of this process.

\section{Introduction}

Post-intubation tracheal stenosis (PTS) is a progressive and constant narrowing of the tracheal lumen with fibrous scars, and is related to the use of intubation tubes $(1,2)$. PTS is a life-threatening pathological modification of the upper segment of the trachea. The absolute number of PTS cases, which is regarded as the most common cause of benign tracheostenosis, is rising due to the increased use of assisted ventilation in intensive care units. Tracheal stenosis of patients is often caused by prolonged tracheal intubation, which may require surgery to remove the narrowed portion of the airway or endoscopic interventional therapy, to which close attention has been paid in recent years, but these methods can only achieve short-term effects. To date, the mechanism of PTS remains unclear. Certain studies suggest that the nature of scar formation in the tracheal lumen resulting in stenosis shows that PTS is a fibroproliferative disease, which is caused by aberrant repair after tissue injury $(3,4)$. Its pathological hallmark is the overexpression of extracellular matrix (ECM) presenting collagen type I, collagen type III and fibronectin, while elastic fibers was significantly reduced. Fibroblasts, as the main cells synthesizing ECM, play an important role in granulation formation, wound contraction and scar formation. The lethality and increasing morbidity of PTS have led us to investigate the precise processes underlying pathological stenosis in order to develop clinical tools to inhibit scar formation.

Hypoxia may be an important factor in the induction of post-intubation tracheal hypertrophic scars. Studies of tracheal stenosis showed that the pressure exerted by the cuff on the tracheal mucosa is the main cause of mucosal ischemia and anoxia, followed by tracheal mucosal erosion, ulceration and chondritis $(5,6)$. Fibrous scar formation is a compensatory mechanism aimed at healing the circumferential damage. Hypoxia-inducible factor (HIF)-1 has been investigated in recent years, as HIF-1 is a DNA-binding protective nuclear transcription factor under hypoxia in mammalian and human cells, and is associated with specific nuclear cofactors to transactivate genes in order to adapt to the compromised oxygen tension. 
HIF-1 is a heterodimer comprising an active $\alpha$-subunit (HIF-1 $\alpha$ ) and a constitutive $\beta$-subunit, which indicates that the biological activities are determined by the expression of HIF-1 $\alpha$ (7). Under normoxic conditions, HIF-1 $\alpha$ is hydroxylated on proline residues by prolyl-hydroxylase domain (PHDs)-containing enzymes, then recognized by the $\beta$-subunit, and subsequently ubiquitylated and degraded by the $26 \mathrm{~S}$ proteasome (8-10). With lowering oxygen tension in the microenvironment, HIF-1 $\alpha$ is abrogated from hydroxylation as PHDs require oxygen for catalytic activity, and therefore HIF- $1 \alpha$ escapes recognition by ubiquitin ligase, which results in the accumulation of HIF-1 $\alpha$ in the nucleus. Upregulation of HIF-1 $\alpha$ subsequently activates a myriad of downstream genes that protect cells in response to hypoxia. The responses that HIF-1 $\alpha$ mediates in hypoxic conditions include increasing oxygen delivery by the formation of new blood vessels and efficient utilization (11) by the activation of genes, including angiogenesis-related genes, such as vascular endothelial growth factor (VEGF), and genes related to cell proliferation and differentiation, such as transforming growth factor (TGF) and basic fibroblast growth factor (bFGF).

In recent years, increasing evidence has suggested that HIF- $1 \alpha$ acts as a potential contributor to facilitate fibrogenesis by activating fibroblast transdifferentiation into myofibroblasts or enhancing epithelial-to-mesenchymal transition in hypoxia (12-15). HIF-1 $\alpha$ is being focally targeted as a novel cancer therapy, as it allows sustained angiogenesis and tissue metastasis of cancer (16). Scheid et al (17) showed that the upregulation of HIF- $1 \alpha$ in adults may represent a pathway in the pathogenesis of scarring. Based on all of the above findings, we speculate that HIF-1 $\alpha$ may be involved in the process of PTS by activating angiogenesis and cell proliferation. The aim of the present study was to identify the expression of HIF-l $\alpha$ and its target genes in tracheal scars of PTS, analyze their correlation in order to explore new hypotheses in the development of PTS, and further provide a theoretical basis for the theory that HIF-1 inhibitors are a preventive method for PTS.

\section{Materials and methods}

Ethics approval. The investigation and protocols were approved by the Bioethical Committee of the Second Hospital of Hebei Medical University (Shijiazhuang, Hebei, China). All procedures were strictly performed according to the Declaration of Helsinki revised in 1983.

\section{Experiment one}

Patients and methods. All intubated patients in the intensive care units of the Second Hospital of Hebei Medical University (Shijiazhuang, Hebei, China) between March 2010 and June 2012, had the tracheal mucosal pressure exerted by the cuff measured through indirect methods. The detailed steps used are as follows: i) the appropriate endotracheal tube was selected and in vitro intracuff pressure was measured after injection of 1-15 ml of gas; ii) after endotracheal intubation, the cuff was inflated to the smallest value at which no air leaked, the in vivo intracuff pressure was measured and recorded at end-expiration, and the volume of inflated air was recorded. The pressure on the tracheal mucosa was calculated by subtracting the in vitro intracuff pressure from the in vivo intracuff pressure of equal volumes of gas. Intracuff pressure in vivo was measured twice per day until extubation. Pressures were measured using cuff pressure gauges (VBM Medizintechnik GmbH, Einsteinstr.1, 72172 Sulz a.N., Germany). The duration of intubation was also recorded. The recorded number of patients was 1,384 cases, and they were followed for six months or until being diagnosed with PTS. Eighteen patients with dyspnea were demonstrated to have tracheal stenosis by neck computed tomography, and the same number of patients without PTS was randomly selected from the recorded cases. We studied the effects of mucosal pressure exerted by the cuff of the tracheal tube and the duration of intubation on scar formation of patients with PTS, and measured distance from the vocal cord to the stenosed site in order to locate tracheal stenosis.

\section{Experiment two}

Patients and samples. We selected specimens from patients according to the following inclusion and exclusion criteria. Inclusion criteria: i) patients with PTS observed and verified by bronchoscopy; ii) tracheal stenosis was related to previous intubation. Exclusion criteria: i) patients who were $<18$ years old; ii) concomitant disease in the region of tracheal stenosis and; iii) patients who were smokers. Normal control tissue samples were not obtained, as normal tissue barely expresses HIF-1 $\alpha$ according to the literature $(16,18)$, which was verified by our preliminary experiment (data not shown).

The selected 24 patients, showing different degrees of dyspnea within six months of endotracheal intubation removal, were admitted to the Second Hospital of Hebei Medical University (Shijiazhuang, Hebei, China) for bronchoscopic interventional therapy. All patients were Chinese and each patient in the two experiments signed a written informed consent form. The age of the patients varied between 18 and 65 years (15 males and 9 females).

According to endoscopic results, patients were divided into three groups (per group, $n=8$ ), namely, granulation phase group (GRA), the proliferative phase group (PRO) and mature phase group (MAT). The specimens were obtained by biopsy forceps through bronchoscopy from the hyperplastic tissue in the tracheal lumen, rinsed in saline, cut into $\sim 1 \mathrm{x} 1 \mathrm{~mm}$ sections using a scalpel, frozen immediately in liquid nitrogen and stored at $-80^{\circ} \mathrm{C}$ for western blotting.

Western blot analysis. Western blot analyses were performed as follows. Firstly, total protein extraction was performed. The specimen was ground in a container with liquid nitrogen, and put on ice with $1 \mathrm{ml}$ of hypotonic buffer A for $1 \mathrm{~h}$. Then, $80 \mathrm{ml}$ of $10 \%$ Nonidet P-40 (NP-40) solution was added to the homogenates, and the mixture was centrifuged at $14,000 \mathrm{x}$ g for $15 \mathrm{~min}$. The protein concentration was determined by a micro bicinchoninic acid assay (Pierce Chemical Co., Rockford, IL, USA), xSDS buffer [0.2 M Tris- $\mathrm{HCl}$ (pH 6.8), 4\% sodium dodecyl sulphate (SDS), $0.18 \%$ glycerol, $0.02 \% \beta$-mercaptoethanol and bromophenol blue] was added to the supernatant samples and boiled for $5 \mathrm{~min}$. Proteins were separated electrophoretically by $10 \%$ SDS-PAGE and then transferred onto a PVDF membrane. After blocking membranes with Odyssey Blocking buffer (LI-COR Biosciences, Inc., Lincoln, NE, USA), immunoblot analysis was performed by incubation with 1:500 diluted primary antibodies against HIF-1 $\alpha$ (Novus Biologicals, LLC, Littleton, CO, USA), VEGF, bFGF and $\beta$-actin (these three 
Table I. Effects of pressure exerted by the cuff on tracheal mucosa and duration of intubation on scar formation in PTS ( $\mathrm{n}=18$ ).

\begin{tabular}{lccc}
\hline Group & Age (years) & Pressure on mucosa (mmHg) & Duration of intubation (days) \\
\hline PTS & $55.00 \pm 12.26$ & $6.39 \pm 2.23^{\mathrm{a}}$ & $20.06 \pm 9.04^{\mathrm{a}}$ \\
NPTS & $53.44 \pm 11.69$ & $20.33 \pm 1.97$ & $12.22 \pm 3.95$ \\
\hline
\end{tabular}

${ }^{a} \mathrm{P}<0.05$ vs. NPTS group. Values are the means \pm standard deviation. PTS, post-intubation tracheal stenosis; NPTS, no PTS.

reagents were obtained from Santa Cruz Biotechnology, Inc., Santa Cruz, CA, USA), $\alpha$-SMA (Sigma-Aldrich, St. Louis, MO, USA), and antibodies against TGF- $\beta$, type I and type III collagen and GAPDH (these four reagents were obtained from Meilian Bioengineering Institute, Shanghai, China) at $4{ }^{\circ} \mathrm{C}$ overnight. The membranes were washed and incubated for $1 \mathrm{~h}$ with horseradish peroxidase-conjugated secondary antibodies (Santa Cruz Biotechnology, Inc.) at room temperature. Proteins were visualized with an enhanced chemiluminescence kit (Santa Cruz Biotechnology, Inc.).

Statistical analysis. Statistical analysis was performed using SPSS (version, 13.0; SPSS Inc., Chicago, IL, USA). The results are presented as the mean values with standard deviation (SD). Comparisons were made by the two-tailed Student's t-test for independent samples or one-way analysis of variance (ANOVA) followed by the least significant difference test. $\mathrm{P}<0.05$ was considered to indicate a statistically significant difference.

\section{Results}

Results of experiment one.

Effects of pressure exerted by the cuff on tracheal mucosa and duration of intubation on tracheal stenosis. There was no difference in age between the two groups. The tracheal mucosal pressure exerted by the endotracheal tube cuff in the PTS group was significantly higher than that in the group without PTS (NPTS; P<0.05; Table I). The duration of intubation was increased in the PTS group compared with the NPTS group $(\mathrm{P}<0.05$; Table I). The location of tracheal stenosis determined by distance from the vocal cord to the stenosed site was $2.30 \pm 0.22 \mathrm{~cm}$, which was consistent with the site where the cuff exerted pressure on the tracheal wall.

\section{Results of experiment two.}

Using western blot analysis, the present study evaluated the protein expression profiles of HIF-1 $\alpha$ and its target genes VEGF, bFGF and TGF- $\beta$, respectively, in different periods of scar formation in 24 patients with PTS. We also evaluated the ECM in the hyperplasia tissue.

$\alpha$-smooth muscle actin ( $\alpha$-SMA) protein expression in different phases of scar formation in PTS. $\alpha$-SMA is a symbol of fibroblast activation. As shown in Fig. 1, the protein expression of $\alpha$-SMA in different phases of tracheal scar formation were significantly different. $\alpha$-SMA was detected in the granulation phase, and markedly increased further in the proliferative phase compared with that of the granulation phase $(\mathrm{P}<0.05)$. The mature phase showed the lowest levels of expression among the three groups $(\mathrm{P}<0.05)$.

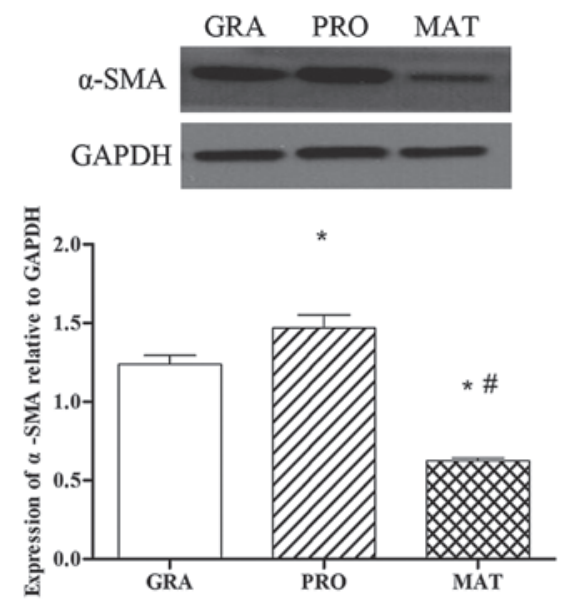

Figure 1. $\alpha$-SMA protein expression in different phases of scar formation were detected by western blot analysis. The bands correspond to $\alpha$-SMA of the GRA, PRO and MAT groups, normalized to that of GAPDH. Values are the means \pm standard deviations, $\mathrm{n}=8$ /group. ${ }^{\text {}} \mathrm{P}<0.05$ vs. GRA; ${ }^{\prime} \mathrm{P}<0.05$ vs. PRO. GRA, granulation phase; PRO, proliferative phase; MAT, mature phase.

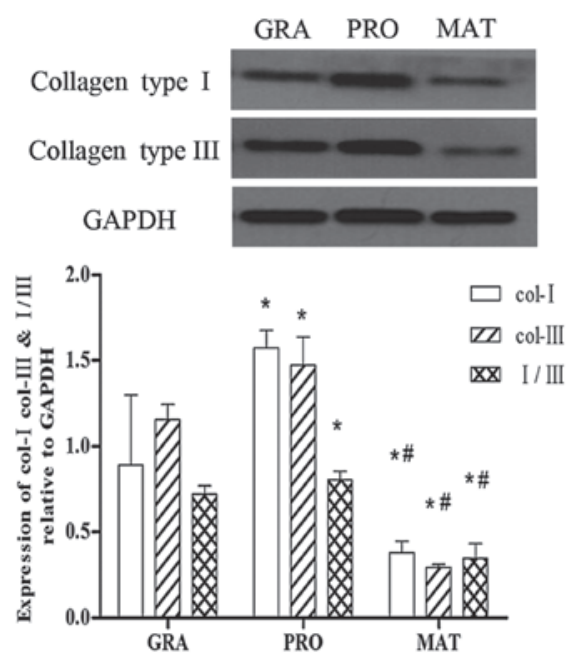

Figure 2. Type I and III collagen protein expression in different phases of scar formation in PTS. The protein expression of type I and III collagen in different phases of scar formation was detected by western blot analysis. The bands correspond to type I and type III collagen of the GRA, PRO and MAT groups. Bar graph shows densitometry of type I and III collagen, normalized to that of GAPDH. Values are the means \pm standard deviations, $n=8 /$ group. ${ }^{*} \mathrm{P}<0.05$ vs. GRA; ${ }^{\mathrm{P}}<0.05$ vs. PRO. GRA, granulation phase; $\mathrm{PRO}$, proliferative phase; MAT, mature phase; PTS, post-intubation tracheal stenosis.

ECM expression in different phases of tracheal scar formation in PTS. Type I and III collagen are the main components of the ECM during scar formation, and are synthesized and secreted 
by myofibroblasts. As shown in Fig. 2, the expression of type I and III collagen in different phases of tracheal scar formation was detected by western blot analysis. Type I and III collagen were increased markedly in the proliferative phase compared with the granulation phase $(\mathrm{P}<0.05)$ and levels were significantly decreased in the mature phase $(\mathrm{P}<0.05)$. The mature phase showed the lowest levels of the three groups. The same trend was observed in the ratio of type I collagen vs. type III collagen.

Protein expression of HIF-1 $\alpha$ and its target genes in different phases of scar formation in PTS. To determine whether the HIF-1 $\alpha$ signaling pathway is involved in the formation of PTS, we investigated protein expression of HIF-1 $\alpha$ and its target genes by western blot analysis. Protein expression of HIF- $1 \alpha$ in the granulation period was the highest of the three groups, and then gradually reduced, so that in the mature stage the levels were significantly reduced compared with the other two stages $(\mathrm{P}<0.05)$ (Fig. 3A and D). Similar trends were observed for protein expression of VEGF (Fig. 3A and B) and bFGF (Fig. 3A and C); whereas for TGF- $\beta$ protein expression, the level of TGF- $\beta$ was highest in the proliferative phase, and there was a statistically significant difference compared with the other two groups $(\mathrm{P}<0.05)$. The mature stage showed the lowest levels of the three groups (Fig. 3A and E).

\section{Discussion}

The present study was performed with the objective of identifying whether molecular pathways induced by hypoxia contribute to the activation of tracheal fibroblasts in PTS. We found in experiment one that the tracheal mucosal pressure exerted by the cuff and the duration of intubation had an effects on PTS. In experiment two, the HIF-1 $\alpha$ protein was markedly expressed in granulation formation phase of PTS, and sustained a high level in the proliferative phase, while it was scarcely detected in the mature phase scars. HIF-1 $\alpha$ target genes related to formation of fibrous scars, such as VEGF and bFGF, were also expressed, whereas the protein expression of TGF- $\beta$ showed a different trend. The ECM proteins showed higher expression in the granulation and proliferative phases compared with that of the mature phase. These results demonstrated that hypoxia contributed to the initiation and progression of fibrosis in PTS and therefore provided a novel hypothesis for the pathogenesis of PTS.

The pathological changes of patients with PTS are similar to those of patients with skin scars, including, but not limited to, fibroblast activation, proliferation, contraction and remodeling of ECM. $\alpha$-SMA, a marker of myofibroblasts, is overexpressed after fibroblasts are activated to form myofibroblasts according to experiments in vivo and in vitro. The activated fibroblasts produce ECM. The two types of collagen, I and III, comprising the main structural element of the ECM during the healing process, play the largest role in wound repair and correlate with the sustained presence of myofibroblasts $(19,20)$, as they present a significant capacity of tension-resistance to form a protective belt following injury. In this study, we observed that $\alpha$-SMA expression in the proliferative group markedly increased (Fig. 1), which suggested transformation of fibroblasts to myofibroblasts, compared with the granulation and mature groups. In turn, myofibroblasts produce large amounts of collagen, therefore collagen type I and type III were observed to be markedly accumulated in the proliferative and granulation phases of the tracheal pathological hyperplasia, particularly in the proliferative phase, and significantly decreased in the mature phase. The trend of their ratios was the same (Fig. 2). Our results revealed that excessive proliferation and active function of myofibroblasts were involved in the repair of tracheal mucosal damage and tracheal stenosis formation in PTS. Our results are in line with previous studies $(21,22)$, in which the investigators reported that tracheal damage promoted production of myofibroblasts and regulated the homeostasis of ECM components.

The clinical investigations revealed that vascular proliferation occurs in the early stage of scar formation. VEGF, as the most important angiogenesis-stimulating factor, plays a pivotal role in the angiogenesis of scars. Le et al (23) revealed a large quantity of VEGF in fibroblasts located in the papillary dermis, and consistent elevation in the homogenate lysate of keloid tissues compared with normal skin controls. Certain studies on airway stenosis have also reported that granulation tissue, as the initial change that occurs in airway stenosis, exhibited an increase in angiogenesis (24-26). Our results were in agreement with these findings. We found that VEGF protein expression in the granulation and proliferative phases significantly increased compared with the mature phase, particularly in the granulation phase (Fig. 3A and B), which suggested the important role of VEGF in tracheal cicatrization. Besides angiogenesis, Wu et al (27) have also indicated that VEGF may alter ECM homeostasis and lead to a state of excessive accumulation and impaired degradation in keloid formation, and Yang et al (28) have shown that VEGF also controlled the dynamics and expression of dermal fibroblasts in wound healing. Taken together, the data suggest that VEGF is important in granulation during tissue repair.

Basic FGF, a member of the fibroblast growth factor family, has been confirmed by an increasing number of studies to promote wound healing by increasing fibroblast migration, proliferation and angiogenic function, and in particular, to play a role in granulation tissue formation (29-31). Accordingly, in the present study we observed that protein expression of bFGF in the granulation phase was the highest of the three phases, and it gradually decreased in the proliferative phase (Fig. 3A and $\mathrm{C}$ ), and there was a marked reduction in the mature phase, which suggests the importance of bFGF in the formation of tracheal stenosis found in PTS.

The emerging experiments showed stabilization and accumulation of HIF- $1 \alpha$ in hypoxia, since its degradation was impeded $(32,33)$. Accumulated HIF-1 $\alpha$ translocates into the nucleus leading to the activation of its target genes related to scar formation, including VEGF and bFGF (35-37). Previous experiments found that HIF-1 $\alpha$ induced myofibroblast differentiation (38) and hypoxia contributes directly to ECM accumulation in patients with systemic sclerosis (39), which suggests that hypoxia may contribute to tracheal fibrosis based on the duration of hypoxia. In experiment one, we found that the pressure exerted on tracheal mucosa by the endotracheal tube cuff was significantly higher in the PTS group than in the NPTS group and the duration of intubation was longer in the PTS group compared with the NPTS group. These results correlated with the conclusions in the studies of Leigh and Maynard (40) and Lewis et al (41), which showed that high tracheal wall pressure that exceeds $20 \mathrm{mmHg}$, which is approximately the mean capillary perfusion pressure, may damage the tracheal mucosa leading 
A

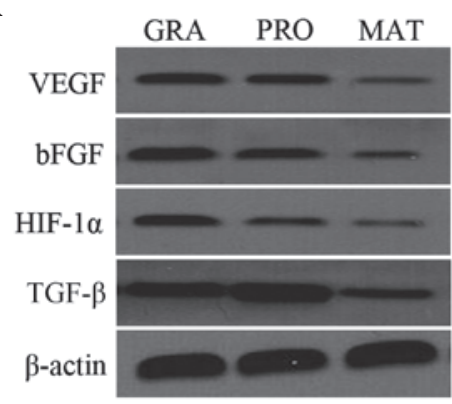

B

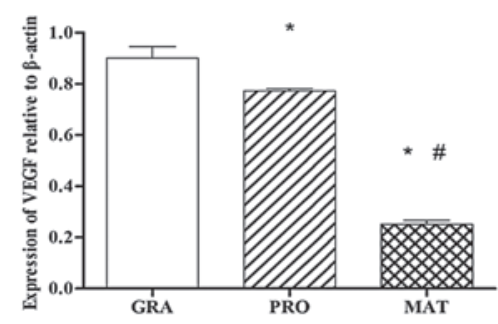

C

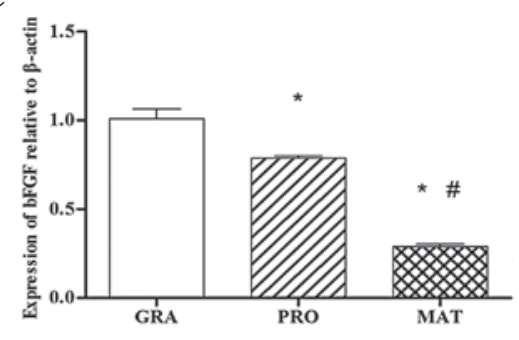

D
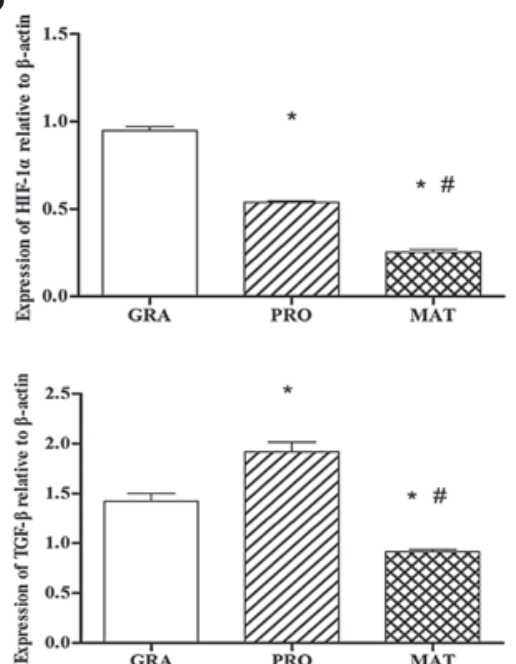

Figure 3. Protein expression of hypoxia-inducible factor-1 $\alpha$ (HIF-1 $\alpha$ ) and its target genes in different phases of scar formation in PTS. Protein expression was detected by western blot analysis. The bands correspond to vascular endothelial growth factor (VEGF), basic fibroblast growth factor (bFGF), HIF-1 $\alpha$, TGF- $\beta$ and $\beta$-actin in the GRA, PRO and MAT groups. Bar graph shows densitometry of VEGF, bFGF, HIF-1 $\alpha$ and TGF- $\beta$, normalized to that of $\beta$-actin. Values are the means \pm standard deviations, $\mathrm{n}=8$ /group. ${ }^{*} \mathrm{P}<0.05$ vs. GRA; ${ }^{*} \mathrm{P}<0.05$ vs. PRO. GRA, granulation phase; PRO, proliferative phase; MAT, mature phase; PTS, post-intubation tracheal stenosis; TGF, transforming growth factor.

to topical hypoxia, particularly when accompanied by prolonged tracheal intubation, and this was the cause of PTS. In the process of scar formation, ECM protein accumulation worsens the hypoxia of interstitial fibroblasts by further increasing the distance to blood vessels, resulting in a vicious circle of ECM accumulation and hypoxia, and therefore the hypoxic microenvironment is sustained. To determine the molecular mechanism involved in the process of PTS, in experiment two, we detected protein expression of HIF-1 $\alpha$. As the HIF-1 $\alpha$ subunit is rapidly degraded and scarcely detected in normoxia $(42,43)$, we studied the pathological state in PTS patients. We found that the protein expression level of HIF-1 $\alpha$ in the granulation phase was the highest among the three groups. HIF-1 $\alpha$ expression was sustained in the proliferative phase, and significantly decreased in the mature scars of patients (Fig. 3A and D). We also observed that the variation of VEGF and bFGF, as two main target genes of HIF-1 $\alpha$, was consistent with that of HIF-1 $\alpha$.

TGF- $\beta$ is well known as a traditional profibrotic factor, and has been identified as the major stimulating factor to promote scar formation. A large number of experiments have shown that sustained expression of TGF- $\beta$ plays an important role in tissue repair, remodelling and hypertrophic scar formation (44-46), as well as in the formation of stent-related airway stenosis (47). Previous studies found that TGF- $\beta$, as one of the HIF-1 $\alpha$ target genes, was regulated by HIF-1 $\alpha(48,49)$. In this experiment, we observed that in tracheal scars of PTS patients, HIF- $1 \alpha$ and TGF- $\beta$ protein expression in the granula- tion phase and proliferative phase were significantly higher than the mature phase, but the peak TGF- $\beta$ levels occurred in the proliferative phase (Fig. 3A and E), which was not in accordance with HIF-1 $\alpha$. The possible reasons leading to this discrepancy may be based on cell type, extent and duration of hypoxic state (11) or some other uncertain cause. This result indicates the mechanism of PTS is more complex and involves a network in vivo. Other pathways or genes (50) may act on the TGF- $\beta$ pathway of fibration besides HIF- $1 \alpha$ regulation.

Based on our results, we concluded that ischemia and hypoxia of the tracheal wall on which the cuff exerted the pressure leads to stabilization and an increase of HIF-1 $\alpha$ levels. HIF-1 $\alpha$ subsequently induced overexpression of its target genes related to fibroplastic events. Corresponding to these target genes, fibroblasts were activated in impaired trachea resulting in the persistent and abundant proliferation of myofibroblasts. This study serves as the first part of efforts to examine HIF-1 $\alpha$ as a possible leading regulator in the process of PTS. Our group will perform further in vitro and animal studies in order to explore the cellular and molecular mechanisms involved and to identify whether a HIF-1 $\alpha$ inhibitor could be used as an adjuvant medicine for the treatment of PTS.

\section{Acknowledgements}

This study was supported by grants from the Medical Research Foundation of the Hebei Province (no. 20120064). 


\section{References}

1. Freitag L, Ernst A, Unger M, Kovitz K and Marquette CH: A proposed classification system of central airway stenosis. Eur Respir J 30: 7-12, 2007.

2. Wynn TA: Cellular and molecular mechanisms of fibrosis. J Pathol 214: 199-210, 2008.

3. Dohar JE, Klein EC, Betsch JL, et al: Acquired subglottic stenosis-depth and not extent of the insult is key. Int J Pediatr Otorhinolaryngol 46: 159-170, 1998

4. Corrêa Reis JG, Takiya CM, Lima Carvalho A, et al: Myofibroblast persistence and collagen type I accumulation in the human stenotictrachea. Head Neck 34: 1283-1293, 2012.

5. Knowlson GT and Bassett HF: The pressures exerted on the trachea by endotracheal inflatable cuffs. Br J Anaesth 42 : 834-837, 1970.

6. Cooper JD and Grillo HC: The evolution of tracheal injury due to ventilatory assistance through cuffed tubes: a pathologic study. Ann Surg 169: 334-348, 1969.

7. Jiang $\mathrm{BH}$, Zheng JZ, Leung SW, Roe R and Semenza GL: Transactivation and inhibitory domains of hypoxia-inducible factor $1 \alpha$. Modulation of transcriptional activity by oxygen tension. J Biol Chem 272: 19253-19260, 1997.

8. Ohh M, Park CW, Ivan M, et al: Ubiquitination of hypoxia-inducible factor requires direct binding to the $\beta$-domain of the von Hippel-Lindau protein. Nat Cell Biol 2: 423-427, 2000.

9. Maxwell PH, Wiesener MS, Chang GW, et al: The tumour suppressor protein VHL targets hypoxia-inducible factors for oxygen-dependent proteolysis. Nature 399: 271-275, 1999.

10. Masson N, Willam C, Maxwell PH, Pugh CW and Ratcliffe PJ: Independent function of two destruction domains in hypoxia-inducible factor- $\alpha$ chains activated by prolyl hydroxylation. EMBO J 20: 5197-5206, 2001.

11. Semenza GL: Regulation of cancer cell metabolism by hypoxia-inducible factor 1. Semin Cancer Biol 19: 12-16, 2009.

12. Norman JT, Clark IM and Garcia PL: Hypoxia promotes fibrogenesis in human renal fibroblasts. Kidney Int 58: 2351-2366, 2000

13. Basu RK, Hubchak S, Hayashida T, Runyan CE, Schumacker PT and Schnaper HW: Interdependence of HIF- $1 \alpha$ and TGF- $\beta /$ Smad3 signaling in normoxic and hypoxic renal epithelial cell collagen expression. Am J Physiol Renal Physiol 300: F898-F905, 2011.

14. Clancy RM, Zheng P, O'Mahony M, et al: Role of hypoxia and cAMP in the transdifferentiation of human fetal cardiac fibroblasts: implications for progression to scarring in autoimmune-associated congenital heart block. Arthritis Rheum 56: 4120-4131, 2007.

15. Higgins DF, Kimura K, Bernhardt WM, et al: Hypoxia promotes fibrogenesis in vivo via HIF-1 stimulation of epithelial-to-mesenchymal transition. J Clin Invest 117: 3810-3820, 2007.

16. Nordgren IK and Tavassoli A: Targeting tumour angiogenesis with small molecule inhibitors of hypoxia inducible factor. Chem Soc Rev 40: 4307-4317, 2011

17. Scheid A, Wenger RH, Christina $\mathrm{H}$, et al: Hypoxia-regulated gene expression in fetal wound regeneration and adult wound repair. Pediatr Surg Int 16: 232-236, 2000.

18. Hiroi M, Mori K, Sakaeda Y, et al: STAT1 represses hypoxiainducible factor-1-mediated transcription. Biochem Biophys Res Commun 387: 806-810, 2009.

19. Sidgwick GP and Bayat A: Extracellular matrix molecules implicated in hypertrophic and keloid scarring. J Eur Acad Dermatol Venereol 26: 141-152, 2012.

20. Friedman DW, Boyd CD, Mackenzie JW, Norton P, Olson RM and Deak SB: Regulation of collagen gene expression in keloids and hypertrophic scars. J Surg Res 55: 214-222, 1993.

21. Bellon G, Moreau M, Cam YJ, et al: Modifications of collagens in the course of inflammatory tracheal stenoses. Ann Otol Rhinol Laryngol 94: 403-408, 1985.

22. Doolin EJ, Tsuno K, Strande LF and Santos MC: Pharmacologic inhibition of collagen in an experimental model of subglottic stenosis. Ann Otol Rhinol Laryngol 107: 275-279, 1998.

23. Le AD, Zhang Q, Wu Y, et al: Elevated vascular endothelial growth factor in keloids: relevance to tissue fibrosis. Cells Tissues Organs 176: 87-94, 2004.

24. Liu H, Chen JC, Holinger LD and Gonzalez-Crussi F: Histopathologic fundamentals of acquired laryngeal stenosis. Pediatr Pathol Lab Med 15: 655-677, 1995.

25. Pokharel RP, Maeda K, Yamamoto T, et al: Expression of vascular endothelial growth factor in exuberant tracheal granulation tissue in children. J Pathol 188: 82-86, 1999.
26. Rahbar R, Brown LF, Folkman J, et al: Role of vascular endothelial growth factor A in children with acquired airway stenosis. Ann Otol Rhinol Laryngol 116: 430-435, 2007.

27. Wu Y, Zhang Q, Ann DK, et al: Increased vascular endothelial growth factor may account for elevated level of plasminogen activator inhibitor-1 via activating ERK1/2 in keloid fibroblasts. Am J Physiol Cell Physiol 286: C905-C912, 2004.

28. Yang GP, Lim IJ, Phan TT, Lorenz HP and Longaker MT: From scarless fetal wounds to keloids: molecular studies in wound healing. Wound Repair Regen 11: 411-418, 2003.

29. Suehiro A, Hirano S, Kishimoto Y, Tateya I, Rousseau B and Ito J: Effects of basic fibroblast growth factor on rat vocal fold fibroblasts. Ann Otol Rhinol Laryngol 119: 690-696, 2010.

30. Barrientos S, Stojadinovic O, Golinko MS, Brem H and Tomic-Canic M: Growth factors and cytokines in wound healing. Wound Repair Regen 16: 585-601, 2008.

31. Powers CJ, McLeskey SW and Wellstein A: Fibroblast growth factors, their receptors and signaling. Endocr Relat Cancer 7: $165-197,2000$

32. Wagatsuma A, Kotake N and Yamada S: Spatial and temporal expression of hypoxia-inducible factor-1 $\alpha$ during myogenesis in vivo and in vitro. Mol Cell Biochem 347: 145-155, 2011.

33. Distler JH, Jüngel A, Pileckyte M, et al: Hypoxia-induced increase in the production of extracellular matri-x proteins in systemic sclerosis. Arthritis Rheum 56: 4203-4215, 2007.

34. Sogabe Y, Abe M, Yokoyama Y and Ishikawa O: Basic fibroblast growth factor stimulates human keratinocyte motility by Rac activation. Wound Repair Regen 14: 457-462, 2006.

35. Brahimi-Horn $\mathrm{C}$ and Pouyssegur J: The role of the hypoxia-inducible factor in tumor metabolism growth and invasion. Bull Cancer 93: E73-E80, 2006.

36. Chang JH, Han KY and Azar DT: Wound healing fibroblasts modulate corneal angiogenic privilege: interplay of basic fibroblast growth factor and matrix metalloproteinases in corneal angiogenesis. Jpn J Ophthalmol 54: 199-205, 2010.

37. Bos R, van Diest PJ, de Jong JS, van der Groep P, van der Valk P and van der Wall E: Hypoxia-inducible factor-1 $\alpha$ is associated with angiogenesis, and expression of bFGF, PDGF-BB, and EGFR in invasive breast cancer. Histopathology 46: 31-36, 2005.

38. Kottmann RM, Kulkarni AA, Smolnycki KA, et al: Lactic acid is elevated in idiopathic pulmonary fibrosis and induces myofibroblast differentiation via $\mathrm{pH}$-dependent activation of transforming growth factor- $\beta$. Am J Respir Crit Care Med 186: 740-751, 2012.

39. Distler JH, Jungel A, Pileckyte M, et al: Hypoxia-induced increase in the production of extracellular matrix proteins in systemic sclerosis. Arthritis Rheum 56: 4203-4215, 2007.

40. Leigh JM and Maynard JP: Pressure on the tracheal mucosa from cuffed tubes. Br Med J 1: 1173-1174, 1979.

41. Lewis FR Jr, Schiobohm RM and Thomas AN: Prevention of complications from prolonged tracheal intubation. Am J Surg 135: 452-457, 1978.

42. Lee JW, Bae SH, Jeong JW, Kim SH and Kim KW: Hypoxia-inducible factor (HIF-1) $\alpha$ : its protein stability and biological functions. Exp Mol Med 36: 1-12, 2004.

43. Powis G and Kirkpatrick L: Hypoxia inducible factor- $1 \alpha$ as a cancer drug target. Mol Cancer Ther 3: 647-654, 2004.

44. Leask A and Abraham DJ: TGF- $\beta$ signaling and the fibrotic response. FASEB J 18: 816-827, 2004

45. Liu W, Wang DR and Cao YL: TGF- $\beta$ : a fibrotic factor in wound scarring and a potential target for anti-scarring gene therapy. Curr Gene Ther 4: 123-136, 2004.

46. Blobe GC, Schiemann WP and Lodish HF: Role of transforming growth factor $\beta$ in human disease. N Engl J Med 342: 1350-1358, 2000.

47. Karagiannidis C, Velehorschi V, Obertrifter B, Macha HN, Linder A and Freitag L: High-level expression of matrix-associated transforming growth factor- $\beta 1$ in benign airway stenosis. Chest 129: 1298-1304, 2006.

48. Higgins DF, Kimura K, Iwano $M$ and Haase VH: Hypoxia-inducible factor signaling in the development of tissue fibrosis. Cell Cycle 7: 1128-1132, 2008.

49. Copple BL: Hypoxia stimulates hepatocyte epithelial to mesenchymal transition by hypoxia-inducible factor and transforming growth factor- $\beta$-dependent mechanisms. Liver Int 30: 669-682, 2010.

50. Chen Z, Zhang D, Yue F, Zheng M, Kovacevic Z and Richardson DR: The iron chelators Dp44mT and DFO inhibit TGF- $\beta$-induced epithelial-mesenchymal transition via up-regulation of N-Myc downstream-regulated gene 1 (NDRG1). J Biol Chem 287: 17016-17028, 2012. 